\title{
Spinal Meningeal Diverticula in Spontaneous Intracranial Hypotension: Analysis of Prevalence and Myelographic Appearance
}

\author{
P.G. Kranz, S.S. Stinnett, K.T. Huang, and L. Gray
}

\begin{abstract}
BACKGROUND AND PURPOSE: Spinal meningeal diverticula have been implicated in the pathogenesis of spontaneous intracranial hypotension and have been proposed as both diagnostic features of and therapeutic targets for the condition. We compared the prevalence and myelographic appearance of spinal diverticula in patients with $\mathrm{SIH}$ and healthy controls.
\end{abstract}

MATERIALS AND METHODS: Patients satisfying the ICHD-2 criteria for SIH were retrospectively identified. CT myelograms of 19 patients with SIH were compared with CT myelograms of 18 control patients. Images were reviewed by 2 blinded neuroradiologists. The prevalence, morphology (round versus multilobulated), size, and location (cervical, upper thoracic, lower thoracic, or lumbar) of spinal meningeal diverticula were analyzed.

RESULTS: There was no difference in the proportion of patients with diverticula in the SIH group compared with the control group (68\% versus $44 \%, P=.14$ ) or in the mean number of diverticula per patient (6.3 versus 2.2, $P=.099$ ). No difference was seen in the morphology $(P=.95)$ or size $(P=.71)$ of diverticula between groups. There was a difference between groups that just reached statistical significance $(P=.050)$ in the location of the diverticula along the spinal axis, but substantial overlap was seen between groups for all spinal locations.

CONCLUSIONS: Despite the well-established association between spinal meningeal diverticula and SIH, we found no difference in the prevalence or myelographic appearance of diverticula in patients with $\mathrm{SIH}$ compared with controls. Further investigation into the role of diverticula in the diagnosis and treatment of $\mathrm{SIH}$ is necessary.

ABBREVIATIONS: $\mathrm{SIH}=$ spontaneous intracranial hypotension; ICHD-2 = International Classification of Headache Disorders, 2nd ed

S pontaneous intracranial hypotension is an increasingly recognized phenomenon characterized by leakage of CSF from the spinal thecal sac. Although the pathogenesis of this disease is not fully understood, diverticula of the spinal nerve roots are thought to represent common sites where CSF leaks originate. ${ }^{1,2}$ Numerous reports have described the presence of large or irregular diverticula in patients with $\mathrm{SIH}$, with some reports offering surgical confirmation of CSF leaking from dural defects localized to meningeal diverticula. ${ }^{3,4}$ In a substantial number of SIH cases, no direct leak is visualized at CT myelography, and meningeal diverticula may be the only myelographic finding (Fig 1).

Received July 12, 2012; accepted after revision August 9.

From the Departments of Radiology (P.G.K., K.T.H., L.G.), Biostatistics and Bioinformatics (S.S.S.), and Ophthalmology (S.S.S.), Duke University Medical Center, Durham, North Carolina.

Please address correspondence to Peter G. Kranz, MD, Department of Radiology, Box 3808, Duke University Medical Center, Durham, NC 27710; e-mail: peter.kranz@duke.edu

http://dx.doi.org/10.3174/ajnr.A3359
Optimal diagnosis and treatment of $\mathrm{SIH}$, including the role of spinal meningeal diverticula in diagnostic and therapeutic algorithms, remain a subject of debate. For example, the presence of spinal diverticula has been proposed as 1 criterion in diagnosing SIH. ${ }^{5}$ Such an approach is not entirely straightforward, however, because diverticula can be observed in asymptomatic patients and no criteria have been described for discriminating normal and abnormal diverticula. ${ }^{6}$ From a therapeutic perspective, surgically exploring or empirically targeting spinal meningeal diverticula with blood or fibrin glue patching has been proposed, ${ }^{5,7}$ an approach that can be challenging when a large number of diverticula are present. It is therefore important to determine whether diverticula present in patients with spontaneous CSF leaks are different from diverticula in normal patients. To our knowledge, no studies have previously compared the prevalence or appearance of spinal meningeal diverticula in patients with SIH and healthy controls.

The purpose of this investigation was to compare the myelographic appearance of spinal nerve root sheaths and associated meningeal diverticula in patients with SIH and healthy controls. 

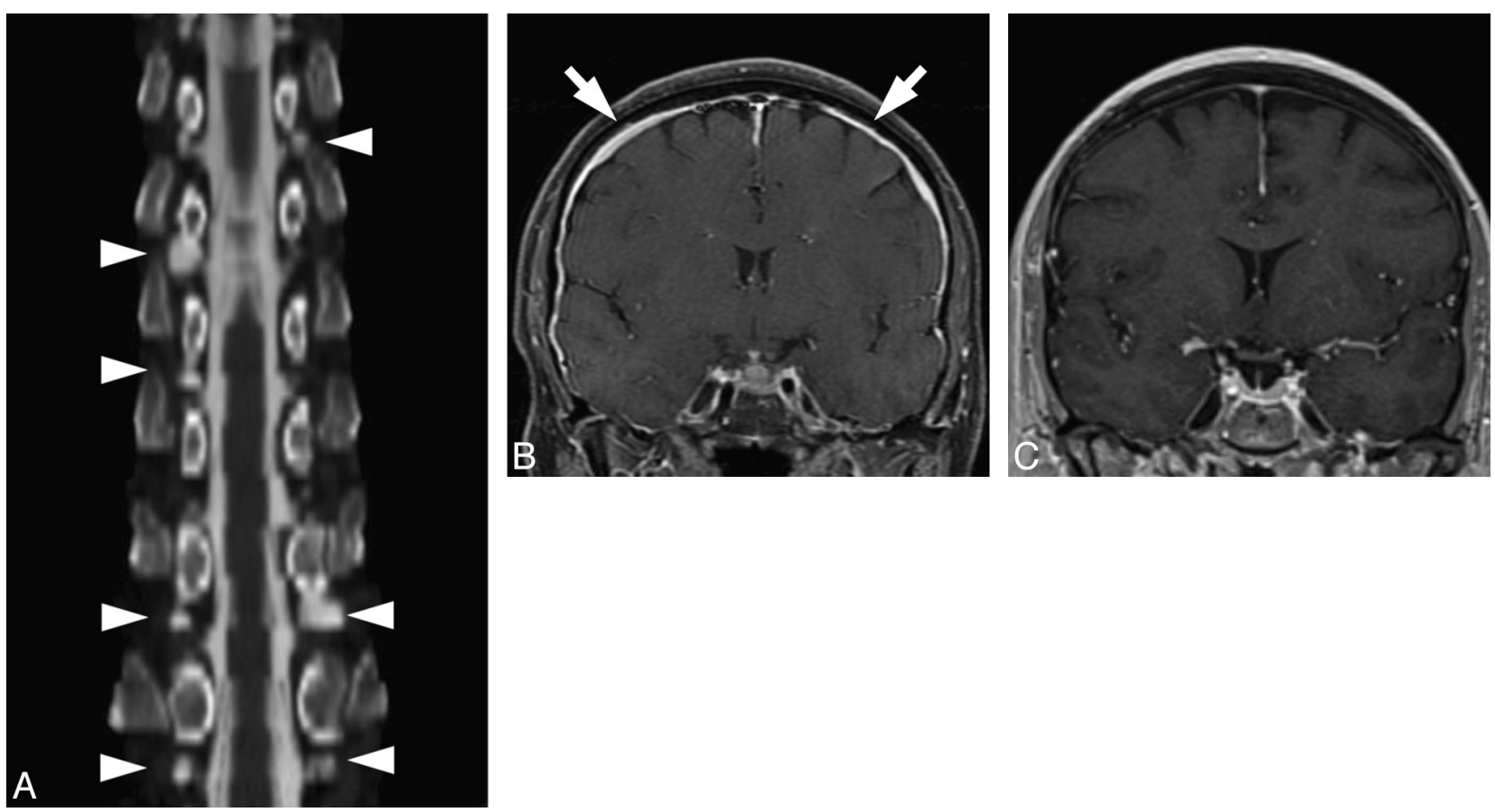

FIG 1. A 58-year-old woman with positional headaches and tinnitus. A, Curved plane reformatted image from a CT myelogram demonstrates numerous spinal meningeal diverticula of varying sizes (arrowheads); no CSF leak was directly visualized. $B$, Coronal T1 postcontrast image from an MR image obtained before treatment shows diffuse smooth dural enhancement (arrows) and pituitary enlargement, compatible with SIH. C, Coronal T1 postcontrast image obtained following blood patching targeting the diverticula seen in $A$ shows resolution of the imaging findings of SIH. The patient's symptoms completely resolved following treatment.

Our hypothesis was that patients with SIH would demonstrate an increased number of diverticula and that diverticula in patients with SIH would more likely be irregular, large, and predominantly located in the upper thoracic spine.

\section{MATERIALS AND METHODS \\ Subjects}

This investigation was a retrospective case control study comparing the CT myelographic appearance of spinal meningeal diverticula in patients with and without SIH. The investigation was approved by the local institutional review board and was Health Insurance Portability and Accountability Act-compliant. A waiver of informed consent was obtained for this retrospective review.

Cases were identified by reviewing the PACS archive for CT myelograms obtained between January 2006 and January 2011, with a clinical indication of headache or suspected intracranial hypotension. Clinical history and brain imaging studies were reviewed in consensus by 2 neuroradiologists. Patients satisfying ICHD-2 criteria for the diagnosis of SIH were included. ${ }^{8}$ Patients with equivocal clinical or imaging findings who failed to satisfy the ICHD-2 criteria were excluded.

Control patients were identified by reviewing the PACS archive for CT myelograms covering the cervical, thoracic, and lumbar spine obtained during the same period, without a clinical indication of headache or SIH. Clinical records of patients in the control group were reviewed to determine the indication for the study and comorbidities. Exclusion criteria for the control group consisted of the following: documented presence of a primary or secondary headache disorder, documented connective tissue dis- order, congenital spinal deformity, technically inadequate myelogram, or a block to contrast that precluded assessment of all imaged levels.

For patients with SIH, lumbar puncture and myelography were performed under CT fluoroscopy, by using the technique previously described by the authors. ${ }^{7}$ For control patients, conventional fluoroscopic guidance was used to inject the myelographic contrast, and the patient was then transported to the CT scanner for imaging. Studies were reviewed on a PACS workstation in consensus by 2 neuroradiologists who were blinded to clinical history. Demographic information was collected from both patient groups.

\section{Image Analysis}

Images were analyzed for the presence of meningeal diverticula occurring at the level of the nerve root sleeve. Each identified diverticulum was classified as round or multilobulated in morphology; representative examples of each morphologic type are depicted in Fig 2. Diverticular size was trichotomized into 3 groups: $0-3,4-6$, and $>6 \mathrm{~mm}$ (Fig 3). Location was categorized as cervical, upper thoracic (T1-T6), lower thoracic (T7-T12), and lumbar. The presence or absence of extrathecal contrast collections around the spine due to rapid active CSF leakage (Fig 4) was also recorded.

We have observed some cases of SIH nerve root sleeves that are of normal tubular contour but show prominent filling on myelography. Because CSF leaks have been described from abnormal "nude" nerve root sleeves and other dural abnormalities of the nerve root sheath in the absence of spinal meningeal diverticula, ${ }^{3}$ we also investigated the prevalence of such "prominent" nerve 

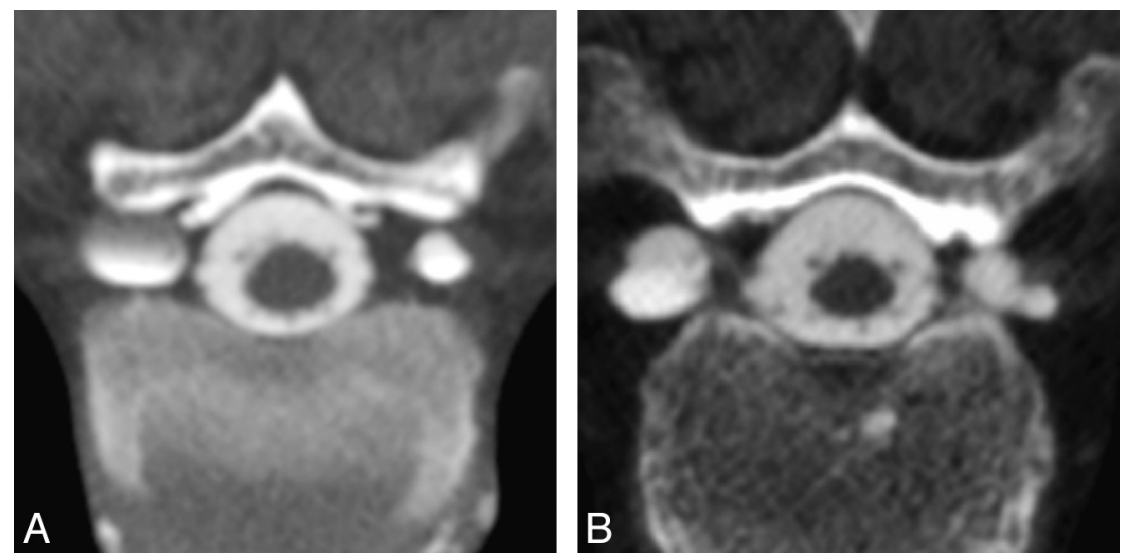

Analysis of diverticular morphology, size, and location is summarized in Table 4. No difference was seen in the relative prevalence of round or multilobulated diverticula in the SIH compared with control group $(P=.95)$ nor was there a difference in the distribution of diverticular sizes between groups $(P=.71)$. Diverticula were found most commonly in the thoracic region, with the lower thoracic region representing the largest single subgroup in both the $\mathrm{SIH}$ and control groups. There was a difference in location between the groups that just reached statistical significance $(P=.05)$, with slightly higher proportions of diverticula in the upper thoracic and lumbar regions in the SIH group.

root sheaths (Fig 5). Because in our experience, nerve sheath dilation is seen commonly in the lower lumbar and sacral spine in healthy patients but spontaneous CSF leaks are rarely encountered in these locations, ${ }^{9,10}$ we excluded the lower 3 lumbar and sacral levels from analysis.

\section{Statistical Analysis}

Statistical analysis was performed by using SAS (Version 9.2; SAS Institute, Cary, NC). The $\chi^{2}$ test was used to compare differences between groups in sex and the proportion of patients with diverticula present. The Wilcoxon rank sum test was used to compare groups as to patient ages and diverticula per patient based on sex. The Fisher exact test was used to compare groups for the presence and prevalence of prominent nerve root sheaths. Differences between groups in the proportion of diverticular morphology, location, and size were assessed by using the generalized estimating equations test of difference, because these data are clustered or correlated in that they represent multiple diverticula from the same patient. A $P$ value of $<.05$ was considered statistically significant.

\section{RESULTS}

Nineteen patients were included in the SIH group, and 18 patients were included in the control group. Demographic data are summarized in Table 1. There was no difference in patient age between the SIH and control groups $(P=.19)$. There was a greater proportion of female patients in the SIH group compared with the control group (74\% versus 33\%, $P=.014$ ). Analysis of diverticular numbers based on sex, however, showed no difference between men and women (Table 2).

Clinical indications for the myelograms obtained in the control group included musculoskeletal pain $(n=8)$, myelopathy $(n=8)$, suspected cord compression $(n=1)$, and suspected spinal cord herniation $(n=1)$.

Spinal meningeal diverticula were observed in 13/19 patients with SIH and 8/18 controls, a difference that was not statistically significant $(P=.14)$ (Table 3). There was also no difference in the mean number of diverticula per patient between the SIH and control groups (mean diverticula per patient, $9.2 \pm 8.2$ versus $4.9 \pm 3.3 ; P=.099)$.
In the SIH group, an extradural contrast collection indicative of a rapid CSF leak was present on CT myelograms in 10 of the 19 patients (52\%). Among the subgroup of patients with SIH with a rapid CSF leak and extradural contrast collection, there were significantly fewer diverticula per patient compared with those in patients with SIH, in whom no extradural collection was identified (mean diverticula per patient, $1.5 \pm 2.9$ versus $12.8 \pm 8.4 ; P=$ .005). No CSF leaks were seen in the control population.

Prominent nerve root sheaths (ie, nerve root sheaths seen filling with contrast after myelography) were more than twice as common in patients with SIH compared with controls $(P=.004)$, though these prominent nerve root sheaths were seen in both the control and SIH groups (Table 3).

\section{DISCUSSION}

Our investigation revealed no difference in the prevalence of spinal meningeal diverticula in patients with SIH compared with healthy controls. We found no significant differences in the number, size, or morphology of these diverticula between the 2 groups and only minimal differences in the distribution of diverticula along the spinal axis. These results suggest that diverticula are common and cannot be used in isolation to distinguish patients with from those without SIH.

The association between SIH and spinal diverticula is wellestablished, with a large number of surgically and radiologically proven cases of CSF leaks originating from abnormal spinal meningeal diverticula. ${ }^{11,12}$ Our results suggest that that the presence or macroscopic features of these diverticula alone do not account for the propensity to develop CSF leaks, however. Some authors have speculated that connective tissue weakness may contribute to the formation of dural defects and fragile diverticula, which may then rupture spontaneously or with minimal trauma, ${ }^{13,14}$ a hypothesis developed out of the observed association between SIH and genetically mediated connective tissue disorders such as Marfan and Ehlers-Danlos syndromes. ${ }^{15-17}$ Our findings do not contradict this hypothesis; however, the presence of diverticula in healthy control patients suggests that connective tissue disorders are not a prerequisite for the formation of diverticula and that the presence of the diverticula alone is not sufficient to cause spontaneous CSF leaks. Rather, microstructural differences within the 

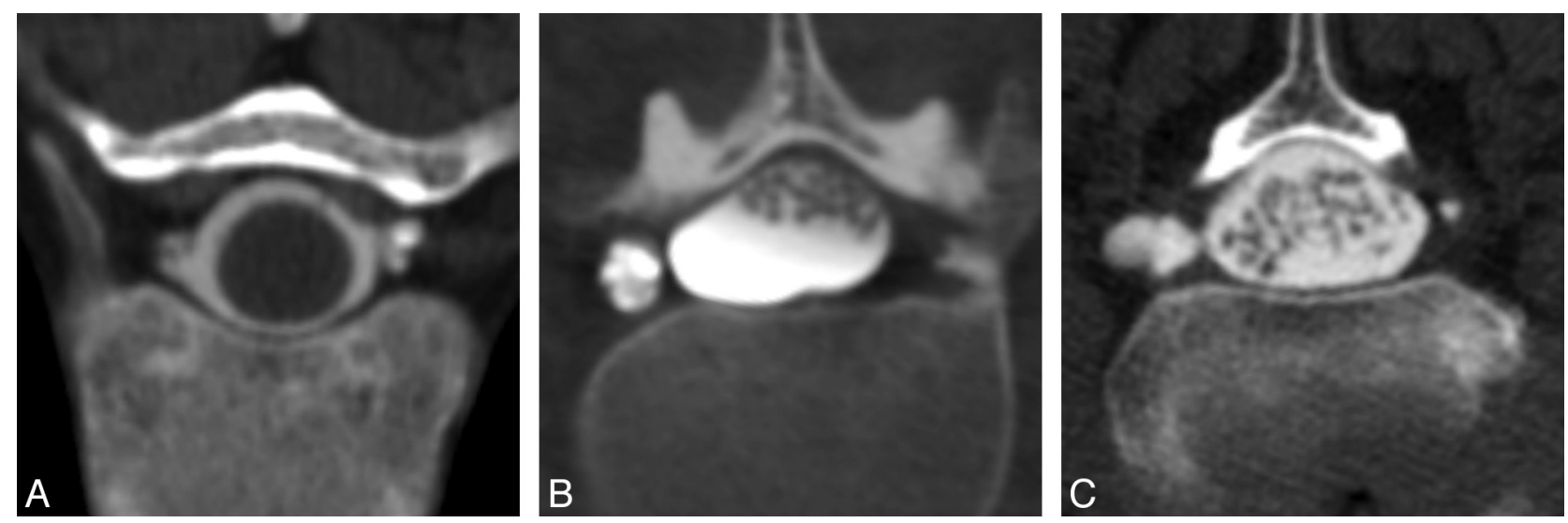

FIG 3. Classification of diverticular size. On the basis of the maximum diameter of the diverticulum on an axial CT myelogram, lesions were trichotomized into 3 groups: $0-3(A), 4-6(B)$, or $>6 \mathrm{~mm}(C)$.

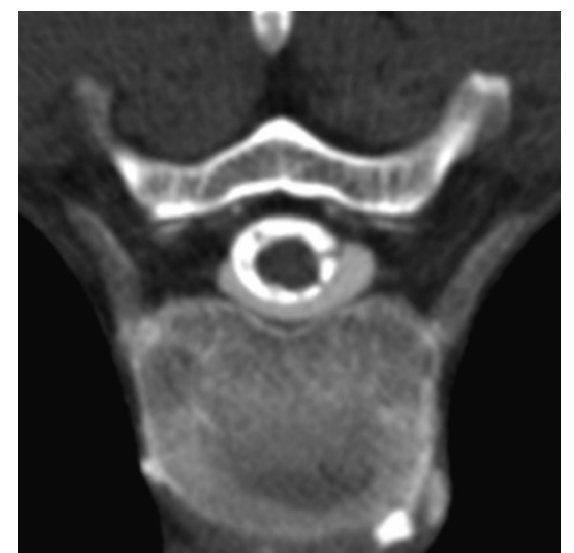

FIG 4. Axial CT myelogram obtained immediately following intrathecal contrast administration in a patient with SIH shows an extrathecal contrast collection. These rapidly filling collections indicate the presence of a high-flow CSF leak.

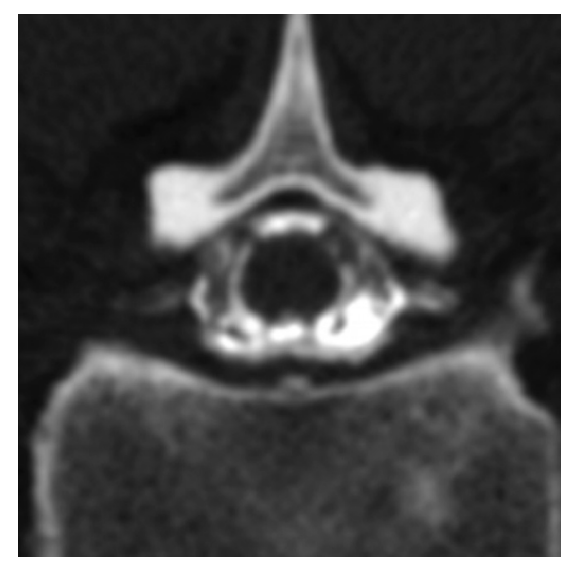

FIG 5. Axial CT myelogram shows filling of the proximal nerve root sheaths bilaterally, findings seen intermittently in both controls and patients with $\mathrm{SIH}$ that we classified as "prominent" nerve root sheaths.

tissue composing the diverticula may play a larger role in CSF leak development than macrostructural features such as size, shape, or location.

Although prominent nerve root sheath filling was seen in both patients with SIH and healthy controls at myelography, this phe-
Table 1: Demographic data

\begin{tabular}{cccc}
\hline & Control & SIH & $\begin{array}{c}\boldsymbol{P} \\
\text { Value }\end{array}$ \\
\hline Age (mean) (SD) (yr) & $55.56(13.85)$ & $50.37(13.27)$ & \\
Min/median/max & $35 / 58 / 77$ & $30 / 52 / 81$ & $.186^{\mathrm{a}}$ \\
Sex & & & \\
Male (No.) (\%) & $12(67)$ & $5(26)$ & \\
Female (No.) (\%) & $6(33)$ & $14(74)$ & $.014^{\mathrm{b}}$ \\
\hline
\end{tabular}

Note:-Min indicates minimum; max, maximum.

a $P$ value based on the Wilcoxon rank sum test of the difference between medians.

${ }^{\mathrm{b}} P$ value based on a $\chi^{2}$ test of the difference in proportions.

Table 2: Prevalence of spinal meningeal diverticula by sex

\begin{tabular}{lccc}
\hline Diverticula per Patient & Male & Female & $\begin{array}{c}\boldsymbol{P} \\
\text { Value }^{\mathrm{a}}\end{array}$ \\
\hline Mean (SD) & $1.6(2.3)$ & $6.5(7.9)$ & .166 \\
Min/median/max & $0 / 1 / 7$ & $0 / 1.5 / 23$ & \\
\hline
\end{tabular}

Note:-Min indicates minimum; max, maximum.

a $P$ value based on the Wilcoxon rank sum test of the difference between medians.

Table 3: Prevalence of spinal meningeal diverticula and prominent nerve sheaths

\begin{tabular}{lccc} 
& Control & SIH & $\begin{array}{c}P \\
\text { Value }\end{array}$ \\
\hline Patients with diverticula present (No.) (\%) & $8(44)$ & $13(68)$ & $.141^{\mathrm{a}}$ \\
$\begin{array}{l}\text { Diverticula per patient (mean) } \\
\quad \text { Min/median/max }\end{array}$ & $2.2(3.3)$ & $6.3(8.0)$ & $.099^{\mathrm{b}}$ \\
$\begin{array}{c}\text { Patients with prominent nerve sheaths } \\
\text { present (No.) (\%) }\end{array}$ & $0 / 0 / 10$ & $0 / 2 / 23$ & \\
$\begin{array}{c}\text { Prominent nerve sheaths per patient } \\
\quad \text { (mean) }\end{array}$ & $2.6(3.1)$ & $6.1(4.2)$ & $.004^{\mathrm{c}}$ \\
$\quad$ Min/median/max & $0 / 1.5 / 13$ & $0 / 5 / 15$ & \\
\hline
\end{tabular}

Note:-Min indicates minimum; max, maximum.

a $P$ value based on a $\chi^{2}$ test of the difference between proportions.

${ }^{b} P$ value based on the Wilcoxon rank sum test of the difference between medians. ${ }^{c} P$ value based on the Fisher exact test of the difference between proportions.

nomenon was observed in a greater number in the SIH group. The significance of this observation remains to be determined. It is possible that an underlying structural or developmental variation could cause a predisposition to both spontaneous CSF leaks and dilation of nerve root sheaths (resulting in their subsequent filling on myelography); however, to our knowledge, there is no direct evidence that confirms this hypothesis.

Our results have implications for both diagnosis and treatment of SIH. The revised International Classification of Headache Disorders criteria currently are the most widely accepted guide- 
Table 4: Comparison of myelographic appearance of spinal meningeal diverticula

\begin{tabular}{lccc}
\hline & Control & SIH & P Value $^{\mathbf{a}}$ \\
\hline Morphology & & & \\
Round (No.) (\%) & $20(51)$ & $62(52)$ & .946 \\
Multilobulated (No.) (\%) & $19(49)$ & $57(48)$ & \\
Size & & & .711 \\
0-3 mm (No.) (\%) & $9(23)$ & $22(18)$ & \\
$4-6 \mathrm{~mm}($ No.) (\%) & $23(59)$ & $26(60)$ & \\
$>6 \mathrm{~mm}($ No.) (\%) & $7(18)$ & & \\
Location & $22)$ & \\
Cervical (No.) (\%) & $6(5)$ & $4(3)$ & \\
Upper thoracic (No.) (\%) & $6(15)$ & $65(28)$ & \\
Lower thoracic (No.) (\%) & $29(75)$ & $17(14)$ & \\
Lumbar (No.) (\%) & $2(5)$ & $55)$ & \\
\hline
\end{tabular}

a $P$ value based on the generalized estimating equations test of the difference between groups in proportions in morphologic categories while accounting for multiple observations per patient.
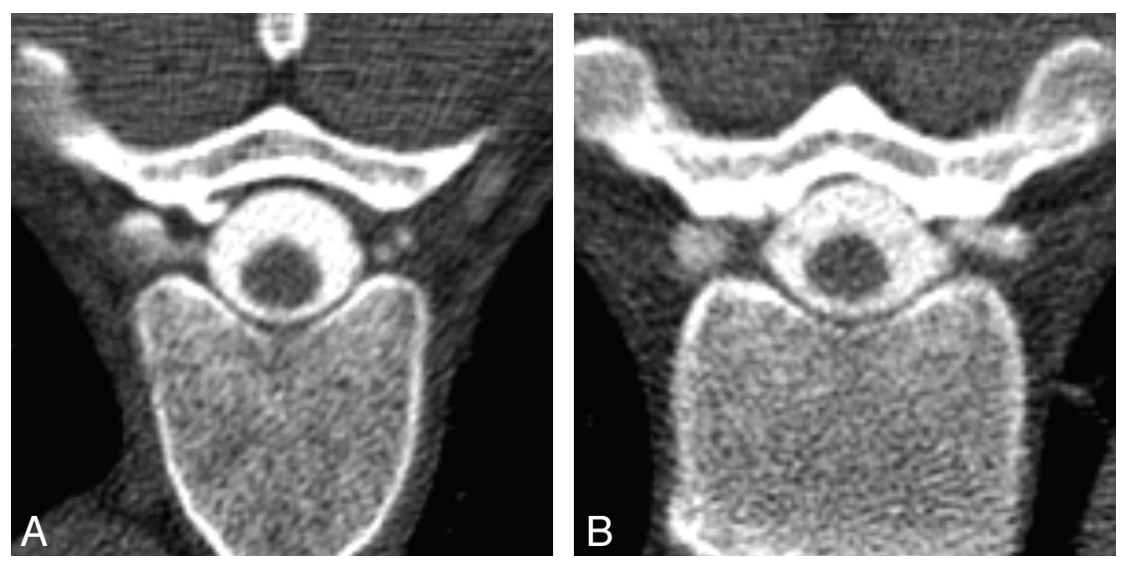

FIG 6. Incidental spinal diverticula in a control patient. Axial CT myelograms demonstrate multiple thoracic spinal diverticula in a 48-year-old woman. The patient underwent the myelography for postoperative back pain; she had no history of headache or other symptoms suggestive of SIH.

lines for establishing the diagnosis of SIH. Nevertheless, there is agreement among many authors that the clinical and imaging features of SIH are more varied than the stereotypical symptoms reflected by the ICHD-2 criteria. ${ }^{18}$ In response to the increased recognition of this diverse spectrum of findings, alternative criteria for diagnosing SIH have been proposed. ${ }^{5}$ These revised criteria propose the use of spinal meningeal diverticula as a criterion for diagnosing SIH, among other factors. While we strongly support the adoption of diagnostic criteria that include the wider spectrum of clinical and imaging findings that can be seen with SIH, our results suggest that using the presence of diverticula as a diagnostic criterion for $\mathrm{SIH}$ would not be justified because they are seen commonly in healthy patients (Fig 6).

With regard to the treatment of $\mathrm{SIH}$, spinal meningeal diverticula have also been proposed for targeted therapy, either via surgical approaches or by using percutaneous blood or fibrin glue patching. ${ }^{7,10,19}$ The use of such a targeted approach is supported by evidence that treating as close as possible to the site of CSF leak is beneficial. ${ }^{9}$ Nevertheless, in a substantial number of cases, imaging does not confirm a definite leak site, presumably due to slow or intermittent CSF leakage. In such patients, it would be useful to predict, from anatomic clues, which diverticula were most likely to be predisposed to leaks. The findings from the present study imply that the myelographic imaging characteristics of any single diverticulum cannot reliably be used for discriminating leaking from nonleaking diverticula when extravasation of CSF is not directly identified. In our opinion, it is likely that new techniques that are more sensitive for detecting small amounts of leaking CSF will have to be developed if optimal treatment of such slow or intermittent CSF leaks is to be achieved.

Interestingly, spinal diverticula were found to be significantly more numerous among patients with SIH and no leak seen on myelography than among their counterparts with SIH in whom a rapid leak was detected. It is possible that this finding could reflect a difference in the etiology of fast leaks compared with slow or intermittent leaks. Alternatively, technical factors may contribute to this observed difference, such as underfilling or underdistension of diverticula in the presence of a rapid leak.

Our investigation has several limitations. First, a limited sample size in this retrospective investigation could potentially result in insufficient power to detect small differences between the 2 groups. However, given the overlap in diverticular characteristics seen in our study, it is doubtful that detection of these small differences would result in clinically useful cutoff values for size, location, or morphology that would allow discrimination of patients with $\mathrm{SIH}$ from controls with a high degree of specificity. In a larger sample, differences might be found among subgroups of patients with different types of CSF leaks, such as those with slow or intermittent leaks, given the increased prevalence of diverticula in this group. Second, CSF leaks have been reported to be caused by etiologies unrelated to the spinal nerve root (such as disk protrusions and osseous spurs). ${ }^{20-22}$ These etiologies could provide alternate mechanisms for causing CSF leak that would not be dependent on meningeal diverticula. We found no imaging evidence of these alternative pathologies in our patient group, however, and think that their impact on our patient sample is likely negligible.

\section{CONCLUSIONS}

Despite the well-established association between spinal meningeal diverticula and spontaneous CSF leakage, our investigation found no differences in the prevalence, number, morphology, or size of spinal diverticula in patients with SIH compared with controls. These findings suggest the need to re-evaluate and further explore the role of spinal meningeal diverticula in the diagnosis and treatment of spontaneous intracranial hypotension. In particular, developing techniques more sensitive for detecting subtle 
CSF leaks and for determining which, if any, diverticula are at risk of leaking would be highly desirable.

\section{REFERENCES}

1. Bai J, Yokoyama K, Kinuya S, et al. Radionuclide cisternography in intracranial hypotension syndrome. Ann Nucl Med 2002;16:75-78

2. Schievink WI, Meyer FB, Atkinson JL, et al. Spontaneous spinal cerebrospinal fluid leaks and intracranial hypotension. J Neurosurg 1996;84:598-605

3. Schievink WI, Jacques L. Recurrent spontaneous spinal cerebrospinal fluid leak associated with "nude nerve root" syndrome: case report. Neurosurgery 2003;53:1216-18, discussion 1218-19

4. Schievink WI, Morreale VM, Atkinson JL, et al. Surgical treatment of spontaneous spinal cerebrospinal fluid leaks. J Neurosurg 1998;88: $243-46$

5. Schievink WI, Maya MM, Louy C, et al. Diagnostic criteria for spontaneous spinal CSF leaks and intracranial hypotension. AJNR Am J Neuroradiol 2008;29:853-56

6. Albayram S, Ozer H, Kara B. Diagnostic criteria for spontaneous spinal CSF leaks and intracranial hypotension. AJNR Am J Neuroradiol 2008;29:e94, author reply e85

7. Kranz PG, Gray L, Taylor JN. CT-guided epidural blood patching of directly observed or potential leak sites for the targeted treatment of spontaneous intracranial hypotension. AJNR Am J Neuroradiol 2011;32:832-38

8. Headache Classification Subcommittee of the International Headache Society. The International Classification of Headache Disorders. 2nd edition. Oxford, UK: Blackwell Publishing; 2004

9. Sencakova D, Mokri B, McClelland RL. The efficacy of epidural blood patch in spontaneous CSF leaks. Neurology 2001;57:1921-23

10. Schievink WI. Spontaneous spinal cerebrospinal fluid leaks and intracranial hypotension. JAMA 2006;295:2286-96

11. Cohen-Gadol AA, Mokri B, Piepgras DG, et al. Surgical anatomy of dural defects in spontaneous spinal cerebrospinal fluid leaks. Neurosurgery 2006;58(4 suppl 2):ONS-238-45, discussion ONS-245

12. Chiapparini L, Farina L, D’Incerti L, et al. Spinal radiological findings in nine patients with spontaneous intracranial hypotension. Neuroradiology 2002;44:143-50, discussion 151-52

13. Schievink WI, Gordon OK, Tourje J. Connective tissue disorders with spontaneous spinal cerebrospinal fluid leaks and intracranial hypotension: a prospective study. Neurosurgery 2004;54:65-70, discussion 70-71

14. Mokri B, Maher CO, Sencakova D. Spontaneous CSF leaks: underlying disorder of connective tissue. Neurology 2002;58:814-16

15. Milledge JT, Ades LC, Cooper MG, et al. Severe spontaneous intracranial hypotension and Marfan syndrome in an adolescent. $J$ Paediatr Child Health 2005;41:68-71

16. Rosser T, Finkel J, Vezina G, et al. Postural headache in a child with Marfan syndrome: case report and review of the literature. J Child Neurol 2005;20:153-55

17. Albayram S, Bas A, Ozer H, et al. Spontaneous intracranial hypotension syndrome in a patient with Marfan syndrome and autosomal dominant polycystic kidney disease. Headache 2008;48:632-36

18. Mokri B. Spontaneous cerebrospinal fluid leaks: from intracranial hypotension to cerebrospinal fluid hypovolemia-evolution of a concept. Mayo Clin Proc 1999;74:1113-23

19. Schievink WI, Reimer R, Folger WN. Surgical treatment of spontaneous intracranial hypotension associated with a spinal arachnoid diverticulum: case report. J Neurosurg 1994;80:736-39

20. Binder DK, Sarkissian V, Dillon WP, et al. Spontaneous intracranial hypotension associated with transdural thoracic osteophyte reversed by primary dural repair: case report. J Neurosurg Spine 2005;2:614-18

21. Winter SC, Maartens NF, Anslow P, et al. Spontaneous intracranial hypotension due to thoracic disc herniation: case report. J Neurosurg 2002;96:343-45

22. Rapport RL, Hillier D, Scearce T, et al. Spontaneous intracranial hypotension from intradural thoracic disc herniation: case report. J Neurosurg 2003;98:282-84 